\title{
Impact de l'hygiène bucco-dentaire sur le sport. Revue de la littérature
}

\author{
Impact of oral hygiene on sport. Review of the literature \\ L. Kipgen ${ }^{\mathrm{a}, *}$, J.-F. Kaux ${ }^{\mathrm{b}}$, E. Rompen ${ }^{\mathrm{c}}$, F. Hérion ${ }^{\mathrm{c}}$ \\ ${ }^{a}$ Centre dentaire de l'Air Pur (CDAP), 20, rue Tige-Manchère, 4120 Rotheux-Rimière, Belgique \\ ${ }^{\mathrm{b}}$ Service de médecine physique, département des sciences de la motricité, université de Liège, CHU de Liège, 4000 Liège, Belgique \\ ${ }^{\mathrm{c}}$ Département de parodontologie et de chirurgie bucco-dentaire, université de Liège, CHU de Liège, 4000 Liège, Belgique
}

Disponible sur Internet le 24 novembre 2014

\begin{abstract}
Résumé
Les dents font l'objet de multiples attentions et soins, mais il n'en va pas de même du parodonte, leur point d'ancrage et de soutien indispensable. Quoi de plus banal, en effet, qu'un saignement survenant lors du brossage quotidien? Étant non douloureux, il ne déclenche bien souvent aucune inquiétude, ni aucune demande de consultation. Il s'agit pourtant d'un des signes cliniques évidents des parodontopathies qui affectent, à des degrés divers, pratiquement $90 \%$ de la population en France dans toutes les tranches d'âge et causent la perte de 30 à $40 \%$ des dents. Depuis plusieurs décennies, le monde scientifique admet une relation entre la maladie parodontale et certaines maladies systémiques, comme l'endocardite, l'athérosclérose, le diabète, l'arthrite rhumatoïde et les naissances prématurées. Et qu'en est-il des tendinopathies ? Leur survenue est souvent spontanée sans origine médicale spécifique et leur guérison s'avère longue et pénible. Cette revue a comme objectif de mettre en évidence les relations entre l'hygiène bucco-dentaire et ses répercussions sur les sportifs.
\end{abstract}

(C) 2014 Elsevier Masson SAS. Tous droits réservés.

Mots clés : Parodontopathies ; Bactérémie ; Tendinopathie

\begin{abstract}
Teeth are the object of multiple attentions and care but unfortunately, it is not the same case for the periodont, their point of anchoring and essential support. What could be more commonplace than a bleeding arising during the daily brushing? Being not painful, it activates very often no concern and no request of consultation. It is nevertheless about one of the most obvious clinical signs of the periodontal diseases which affect, to varying degrees, practically $90 \%$ of the population in France in all groups of ages and are responsible for the loss of 30 to $40 \%$ of the teeth. For several decades, the scientific world admits a relation between the periodontal disease and certain systemic diseases as endocarditis, atherosclerosis, diabetes, rheumatoid arthritis and preterm birth. And what about tendinopathies? Their appearance is often spontaneous without specific medical origin and their cure turns out long and painful. This review has for objective to highlight the relations between oral hygiene and sportsmen.
\end{abstract}

(C) 2014 Elsevier Masson SAS. All rights reserved.

Keywords: Parodontopathies; Bacteremia; Tendinopathie

\section{Introduction}

Les parodontopathies représentent un ensemble de maladies touchant les tissus de soutien des dents [1]. Elles sont

\footnotetext{
* Auteur correspondant.

Adresses e-mail : laurence.kipgen@gmail.com (L. Kipgen), jfkaux@chu.ulg.ac.be (J.-F. Kaux).
}

déclenchées par un biofilm bactérien et se développent, en règle générale, à partir d'une gingivite. La quantité et surtout la virulence des micro-organismes d'une part, et la réponse de l'hôte d'autre part, sont décisives pour le déclenchement et la progression de la destruction parodontale. La gingivite et la parodontite sont toutes les deux responsables d'une libération de médiateurs pro-inflammatoires systémiques [2].

L'Organisation mondiale de la santé (OMS) a déclaré [3] que les maladies bucco-dentaires telles que les caries dentaires et les 
parodontopathies posaient un problème de santé mondial. Globalement, 90 à $95 \%$ de la population montrent des signes de gingivite. Cette prévalence est présente chez l'adulte ainsi que chez les enfants. Chez l'adulte, les premiers stades de parodontopathie sont observés dans 40 à $60 \%$ des cas à partir de 35 à 44 ans. La parodontite sévère touche 5 à $15 \%$ des populations. Une étude de Kulekci et al. [4] a mis en évidence la présence de bactéries pathogènes, comme les Prevotella nigrescens, Treponema denticola, Actinobacillus actinomycetemcomitans (A.a) et Porphyromonas gingivalis (P.g), même chez des enfants entre 6 et 13 ans ne présentant aucun signe clinique d'une quelconque maladie parodontale. Les auteurs recommandent ainsi des dépistages parodontaux préventifs en denture mixte dès le jeune âge chez les enfants à risque. Parahitiyawa et al. [2] ont mis au point une revue de littérature sur le sujet de l'endocardite. Ils décrivent les différentes entrées systémiques possibles en commençant par les lésions carieuses intrapulpaires qui permettent le passage bactérien à travers la chambre pulpaire jusqu'à l'apex dentaire. Toute réaction apicale peut ainsi être responsable d'une bactériémie et nécessite donc, chez des patients à risque, une antibiothérapie préventive lors de soins dentaires (prévention d'Osler) [5-7].

Depuis plusieurs décennies, le monde scientifique admet une relation entre la maladie parodontale et certaines maladies systémiques [8-13], comme l'endocardite [2], l'athérosclérose [14-18], le diabète [19,20], l'arthrite rhumatoïde [16,21] et les naissances prématurées [22]. Malgré une multitude d'études scientifiques, la physiopathologie précise que ces différentes relations entre les maladies parodontales et les maladies systémiques ne sont pas encore élucidées. Il pourrait également exister une relation étiologique entre les parodontites et les tendinopathies [23].

\section{Athlètes et hygiène dentaire}

On s'imagine souvent qu'un sportif de haut niveau est en bonne santé. Le fait que la plupart d'entre eux soit encadrée par des préparateurs physiques, des kinésithérapeutes, des diététiciens et des médecins renforce cette croyance et pourtant, certains travaux mettent en évidence une réalité bien différente.

Une étude dentaire a été menée lors des Jeux olympiques et para-olympiques d'Athènes en 2004, afin d'évaluer et d'améliorer la qualité des soins dentaires chez les sportifs et leur encadrement en vue des Olympiades de 2008. Durant les 17 jours, plus de 870 patients ont été accueillis en dentisterie. Mille six cent quarante soins dentaires ont été réalisés dont 386 obturations, 112 traitements endodontiques, 57 confections de protège-dents, 21 extractions et la prise en charge de 11 traumatismes [24]. Cette étude a mis ainsi en évidence une négligence apparente de la santé dentaire parmi certains athlètes confirmés.

Un travail sur la santé bucco-dentaire a été réalisé en 2011 chez 30 footballeurs professionnels du F.C. Barcelone [25]. Malgré un suivi médical intensif, de nombreux joueurs présentaient des problèmes dentaires comme des caries non traitées, des gingivites et des interférences occlusales. Vingt pourcent des joueurs n'ont pas été chez le dentiste depuis les cinq dernières années et $60 \%$ présentaient régulièrement des saignements gingivaux. Lors de cette étude, les investigateurs ont même mis en évidence une corrélation statistiquement significative entre l'indice de plaque, ainsi que le sondage parodontal et les blessures musculaires des joueurs. L'incidence moyenne de blessures quelconques était de 8 par joueur et par an, dont la majorité est survenue en dehors des entraînements, durant des matchs officiels. Ces études montrent bien que, malgré un suivi médical pointu et sophistiqué, un sportif de haut niveau n'est pas nécessairement mieux averti des problèmes dentaires que toute autre personne, bien au contraire.

\section{Les maladies parodontales et leurs répercussions systémiques [26]}

Les parodontopathies les plus connues sont les gingivites associées à la plaque dentaire (inflammations de la gencive, sans perte d'attache) et les parodontites (destruction des tissus de soutien dentaire due à une inflammation, avec perte d'attache). La gingivite se limite aux tissus mous marginaux et supracrestaux. Elle se manifeste cliniquement par un saignement lors du sondage ainsi que par des rougeurs et un œdème (état avancé), en particulier au niveau des papilles (gencive interdentaire).

Avec des défenses immunitaires réduites, la présence de facteurs de risques, de médiateurs pro-inflammatoires et de bactéries pathogènes pour le parodonte, la gingivite peut évoluer en parodontite. L'inflammation de la gencive atteint alors les structures plus profondes du parodonte et provoque la destruction du collagène et la lyse de l'os (perte d'attache). L'épithélium jonctionnel se transforme en «épithélium de poche» qui migre apicalement et latéralement. Il en résulte l'apparition d'une poche parodontale qui représente alors un refuge et un réservoir pour les bactéries pathogènes opportunistes qui entretiennent et favorisent la progression de la maladie. La plupart des parodontopathies sont des maladies inflammatoires chroniques qui peuvent s'installer pendant des années, sans évoluer. La gingivite est réversible après l'instauration d'une bonne hygiène buccale et l'élimination professionnelle de la plaque et du tartre, tandis que la parodontite qui se développe, en général, à partir d'une gingivite plus ou moins étendue n'est pas totalement réversible.

La progression de la maladie parodontale est en moyenne très lente, mais elle varie fortement selon les individus. Cette différence de vitesse dépend principalement de:

- l'état de santé générale du patient;

- la quantité et la composition de la plaque bactérienne ;

- du statut immunitaire déterminé génétiquement;

- l'appartenance ethnique ;

- l'environnement social ;

- certains facteurs de risque comme le tabagisme et le stress $[27,28]$.

Tous ces éléments sont responsables de la grande diversité des âges d'apparition de la parodontite et des différences dans la vitesse et l'agressivité de son développement (Fig. 1). C'est donc l'environnement de la poche qui, en étant favorable à la prolifération et à la survie de germes pathogènes, permet à ces 


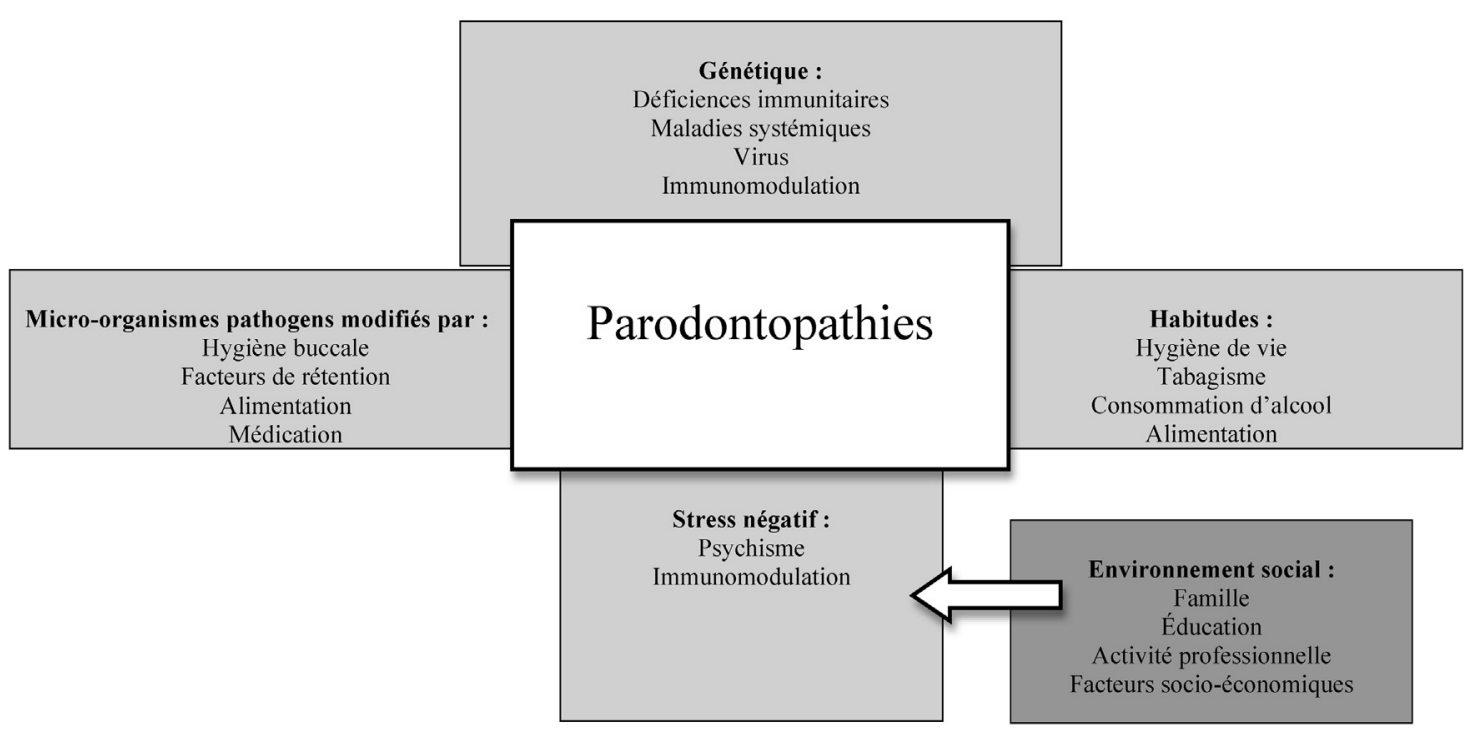

Fig. 1. Étiologie de la parodontite - Interaction entre la plaque dentaire et l'hôte [26].

derniers de faire progresser la maladie et, dans les cas graves, en particulier non traités, peut entraîner la perte de dents.

Lors de la phase inflammatoire, il y a prolifération et dilatation vasculaire induisant une augmentation de la surface d'entrée pour les bactéries.

Toute interruption de l'harmonie entre le biofilm bactérien et les tissus gingivaux conduit à une dissémination bactérienne dans la circulation sanguine. Toute pathologie inflammatoire dentaire, comme les gingivites, parodontites, pulpites ou infections intracanalaires, est à l'origine d'une bactériémie [29] induite par une irritation du parodonte, même de faible intensité.

Une multitude d'études ont démontré que la simple mastication, le sondage parodontal, le surfaçage radiculaire, le dépassement instrumental lors d'un traitement endodontique, les extractions, le brossage et le passage du fil dentaire induisent un passage de bactéries dans la voie sanguine lorsque les tissus gingivaux sont fragilisés par une inflammation [30-33]. Geerts et al. [34] ont mis en évidence la libération systémique d'endotoxines bactériennes d'origine orale suite à la mastication, surtout en cas d'une parodontite sévère. Plus la quantité de plaque accumulée est élevée, plus le passage des bactéries est important. Ces bactériémies sont le plus souvent transitoires et de courte durée avec une intensité maximale à 30 minutes post-traumatisme [2]. Elles permettent à des micro-organismes de se déposer au niveau de certains organes cibles en provoquant des infections sub-cliniques aiguës ou chroniques [13,35]. L'endocardite bactérienne, déclenchée par une extraction dentaire chez un patient à risque, est la complication la plus connue.

Kesavalu et al. [36] ont démontré sur un modèle in vivo que lorsque des micro-organismes oraux, comme P.g et A.a, passent dans les tissus de l'hôte, suite à une bactériémie, ils déclenchent la production de cytokines pro-inflammatoires comme le TNF$\alpha$, IL-1 $\beta$ et l'IL-6. L'intensité de cette réaction dépend du type de bactérie. P.g provoque ainsi une libération de cytokines plus importante que A.a. Il est également important de constater que même des bactéries commensales non pathogènes, comme Streptococcus gordonii, stimulent modérément l'expression de cytokines pro-inflammatoires lors de leur passage dans les tissus de l'hôte. Nilsson et Kopp [37] ont mis en évidence des taux augmentés de TNF circulants, en présence d'une gingivite ou d'une parodontite, chez des patients présentant de l'arthrite rhumatoïde et ayant des indices BOP (Bleeding on Probing), CAL (Clinical Attachement Loss) et PD (Pocket Depth) élevés.

En référence à l'article de Kaux et al. [38], nous avons dressé la Fig. 2 montrant les facteurs de risque communs aux parodontopathies et aux tendinopathies. Ceci a attiré notre attention et nos investigations afin de mettre en évidence l'existence d'une relation éventuelle entre les parodontopathies et les blessures sportives comme des tendinopathies.

\section{Discussion}

Il est communément admis que les parodontopathies sont en interaction forte avec certaines maladies systémiques. Quelques études suspectent également un lien potentiel entre les lésions bucco-dentaires et les blessures chez les sportifs [25]. Lors de notre revue, nous avons pu mettre en évidence l'existence de multiples facteurs étiologiques communs aux parodontopathies et aux tendinopathies (Fig. 2). Serait-il possible que la santé bucco-dentaire ait des interactions concernant la survenue et/ou la guérison des tendinopathies [23] ?

En médecine sportive, les blessures liées à un surentraînement sont évaluées entre 30 et $50 \%$ [38]. La tendinopathie est une des blessures les plus communes résultant d'une surcharge physique $[38,39]$. Elle est la première cause de consultation en médecine sportive et correspond à $30 \%$ des consultations chez le généraliste [38].

De nombreux facteurs étiologiques des parodontopathies peuvent être plus ou moins directement impliqués dans la survenue des tendinopathies [38]. Ils peuvent être répartis en deux grands groupes étiologiques: les facteurs intrinsèques (âge, troubles statiques, dysbalances musculaires, surcharge pondérale, troubles de perfusion, etc.) ou extrinsèques (charges répétitives, progression trop rapide des charges d'entraînement, 


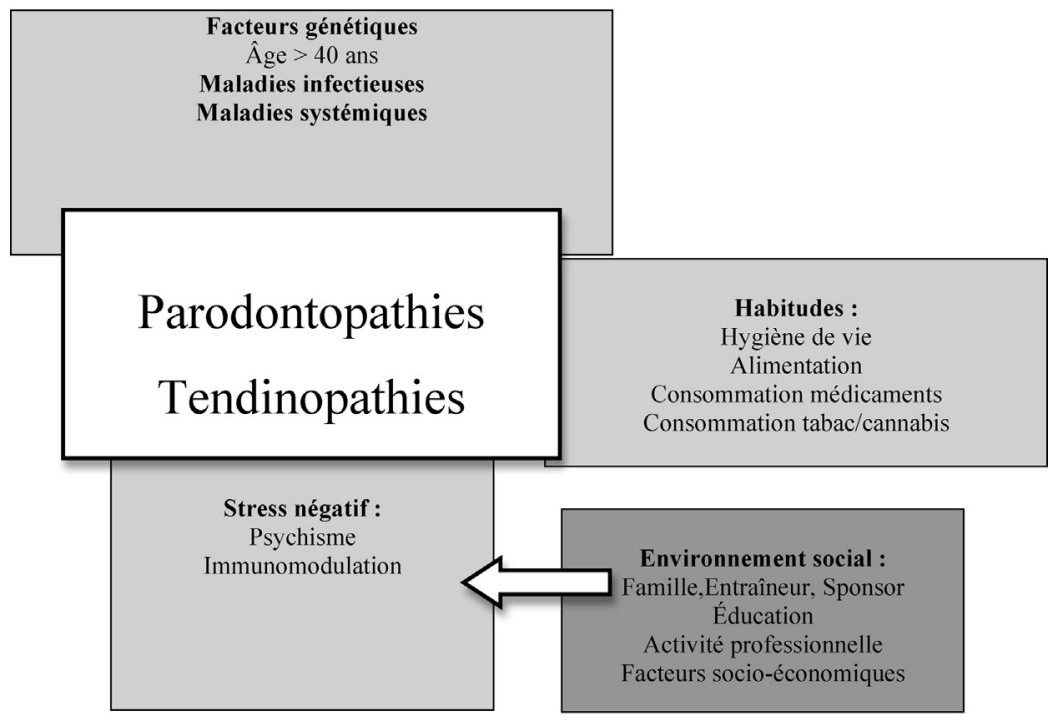

Fig. 2. Facteurs de risques communs aux parodontopathies et aux tendinopathies.

matériel, surface en jeu, geste technique incorrect, etc.) [38]. La prévention des facteurs extrinsèques implique la connaissance du sport incriminé, ce qui rend indispensable un dialogue permanent entre le médecin, le sportif, mais également son entourage (entraîneur, préparateur physique...). Une fois une tendinopathie installée, la guérison s'avère longue et difficile. Bon nombre de sportifs doivent réduire, voire abandonner leur pratique sportive car même les gestes de tous les jours deviennent de plus en plus pénibles. De plus, les tendinopathies peuvent évoluer régulièrement vers la chronicité malgré un traitement conservateur bien conduit [38] et le développement de nouvelles thérapeutiques (ex. plasma riche en plaquettes) [40,41]. En cas de blessure de ce genre, il semble utile d'envisager un simple dépistage parodontal chez le spécialiste et, si nécessaire, la mise au point d'un traitement utile afin de réduire l'influence possible d'une inflammation gingivale sur la guérison sportive.

\section{Conclusion}

La négligence de la santé bucco-dentaire est communément présente dans notre société et conduit à des problèmes majeurs de caries dentaires et de parodontopathies. Malgré l'image du sportif averti et motivé, il se trouve que bon nombre d'athlètes négligent leur santé bucco-dentaire. Les caries sont souvent responsables de gênes et de douleurs, et incitent le patient à la consultation chez le dentiste. L'absence de douleurs dans les parodontopathies les rend peu importantes aux yeux des patients concernés. On sait pourtant que les parodontopathies peuvent agir sur un terrain bien plus profond comme dans l'interaction avec certaines maladies systémiques et, pourquoi pas, lors de la survenue de tendinopathies. Lors d'une carrière sportive, l'apparition d'une blessure comme une tendinopathie entraîne le sportif dans des moments pénibles, longs et douloureux. Elle est souvent accompagnée d'une pause forcée et de traitements médicaux fastidieux.

\section{Déclaration d'intérêts}

Les auteurs déclarent ne pas avoir de conflits d'intérêts en relation avec cet article.

\section{Références}

[1] INSERM. http://lara.inist.fr/bitstream/handle/2332/1389/INSERM Maladies_parodontales_1.pdf

[2] Parahitiyawa NB, Jin LJ, Leung WK, Yam WC, Samaranayake LP. Microbiology of odontogenic bacteremia: beyond endocarditis. Clin Microbiol Rev 2009;22:46-64.

[3] OMS. http://www.who.int/oral_health/media/en/orh_report03_fr.pdf

[4] Kulekci G, Leblebicioglu B, Keskin F, Ciftci S, Badur S. Salivary detection of periodontopathic bacteria in periodontally healthy children. Anaerobe 2008; $14: 49-54$

[5] Somma F, Castagnola R, Bollino D, Marigo L. Oral inflammatory process and general health. Part 2: how does the periapical inflammatory process compromise general health? Eur Rev Med Pharmacol Sci 2011;15:35-51.

[6] Jansma J, Vissink A. Dental foci. Role, treatment and prophylaxis in patients at risk. Ned Tijdschr Tandheelkd 1998;105:52-6.

[7] Debelian GJ, Olsen I, Tronstad L. Bacteremia in conjunction with endodontic therapy. Endod Dent Traumatol 1995;11:142-9.

[8] Lamster IB, DePaola DP, Oppermann RV, Papapanou PN, Wilder RS. The relationship of periodontal disease to diseases and disorders at distant sites: communication to health care professionals and patients. J Am Dent Assoc 2008;139:1389-97.

[9] Martos R, Marton I. Correlations between dental-oral infections and cardiovascular disease. Fogorv Sz 2008;101(3):101-5.

[10] Seymour GJ, Ford PJ, Cullinan MP, Leishman S, Yamazaki K. Relationship between periodontal infections and systemic disease. Clin Microbiol Infect 2007;13(Suppl. 4):3-10.

[11] Costerton J, Keller D. Oral periopathogens and systemic effects. Gen Dent 2007:55:210-5

[12] Cabała A, Chomyszyn-Gajewska M, Drozdz W. Periodontitis and systemic disease relationships. Przegl Lek 2006;63:773-7.

[13] Scannapieco FA. Periodontal inflammation: from gingivitis to systemic disease? Compend Contin Educ Dent 2004;25(7 Suppl. 1):16-25.

[14] Genctoy G, Ozbek M, Avcu N, Kahraman S, Kirkpantur A, Yilmaz $\mathrm{R}$, et al. Gingival health status in renal transplant recipients: relationship between systemic inflammation and atherosclerosis. Int J Clin Pract 2007;61:577-82. 
[15] Lalla E, Lamster IB, Hofmann MA, Bucciarelli L, Jerud AP, Tucker S, et al. Oral infection with a periodontal pathogen accelerates early atherosclerosis in apolipoprotein E-null mice. Arterioscler Thromb Vasc Biol 2003;23:1405-11.

[16] Al-Katma MK1, Bissada NF, Bordeaux JM, Sue J, Askari AD. Control of periodontal infection reduces the severity of active rheumatoid arthritis. J Clin Rheumatol 2007;13:134-7.

[17] Söder B1, Yakob M, Nowak J, Jogestrand T. Risk for the development of atherosclerosis in women with a high amount [corrected] of dental plaque and severe gingival inflammation. Int J Dent Hyg 2007;5:133-8.

[18] Soder B. Dental plaque as a possible risk factor for general disease - results from longitudinal studies. Int J Dent Hyg 2006;(Suppl. 1):22-5.

[19] Lamster IB, Lalla E, Borgnakke WS, Taylor GW. The relationship between oral health and diabetes mellitus. J Am Dent Assoc 2008;139:19S-24S.

[20] Lalla E, Cheng B, Lal S, Tucker S, Greenberg E, Goland R, et al. Periodontal changes in children and adolescents with diabetes: a case-control study. Diabetes Care 2006;29:295-9.

[21] Nilsson M, Kopp S. Gingivitis and periodontitis are related to repeated high levels of circulating tumor necrosis factor-alpha in patients with rheumatoid arthritis. J Periodontol 2009;79:1689-96.

[22] Mitchell-Lewis D, Engebretson SP, Chen J, Lamster IB, Papapanou PN. Periodontal infections and pre-term birth: early findings from a cohort of young minority women in New York. Eur J Oral Sci 2001;109:34-9.

[23] Kipgen L, Kaux JF, Rompen E, Hérion F. Relations entre hygiène buccodentaire et tendinopathies. (soumis).

[24] Vougiouklakis G, Tzoutzas J, Farmakis ET, Farmakis EE, Antoniadou M, Mitsea A. Dental data of the Athens 2004 Olympic and Paralympic Games Int J Sports Med 2008;29:927-33.

[25] Gay-Escoda C, Vieira-Duarte-Pereira DM, Ardèvol J, Pruna R, Fernandez J. Valmaseda-Castellón. Study of the effect of oral health on physical condition of professional soccer players of the Football Club Barcelona. Med Oral Patol Oral Cir Bucal 2011;16:e436-9.

[26] Wolf HF, Rateitschak EM, Rateitschak KH. Parodontologie. Édition Mas son; 2005, ISBN 2-294-01139-2.

[27] Genco RJ1, Ho AW, Grossi SG, Dunford RG, Tedesco LA. Relationship of stress, distress and inadequate coping behaviors to periodontal diseases J Periodontol 1999;70:711-23.
[28] Johannsen A, Bjurshammar N, Gustafsson A. The influence of academic stress on gingival inflammation. Int J Dent Hyg 2010;8:22-7.

[29] Wohlfeil M, Wehner J, Schacher B, Oremek GM, Sauer-Eppe H, Eickholz $\mathrm{P}$. Degree of gingivitis correlates to systemic inflammation parameters. Clin Chim Acta 2009;401:105-9.

[30] Olsen I. Update on bacteraemia related to dental procedures. Transfus Apher Sci 2008;39:173-8.

[31] Kinane DF, Riggio MP, Walker KF, MacKenzie D, Shearer B. Bacteraemia following periodontal procedures. J Clin Periodontol 2005;32:708-13.

[32] Daly CG, Mitchell DH, Highfield JE, Grossberg DE, Stewart D. Bacteremia due to periodontal probing: a clinical and microbiological investigation. J Periodontol 2001;72:210-4.

[33] Forner L, Nielsen CH, Bendtzen K, Larsen T, Holmstrup P. Increased plasma levels of IL-6 in bacteremic periodontitis patients after scaling. J Clin Periodontol 2006;33:724-9.

[34] Geerts SO, Legrand V, Charpentier J, Albert A, Rompen EH. Further evidence of the association between periodontal conditions and coronary artery disease. J Periodontol 2004;75:1274-80.

[35] Scannapieco FA. Systemic effects of periodontal diseases. Dent Clin North Am 2005;49:533-50.

[36] Kesavalu L, Chandrasekar B, Ebersole JL. In vivo induction of proinflammatory cytokines in mouse tissue by Porphyromonas gingivalis and Actinobacillus actinomycetemcomitans. Oral Microbiol Immunol 2002;17:177-80.

[37] Nilsson M, Kopp S. Gingivitis and periodontitis are related to repeated high levels of circulation tumor necrosis factor-alpha in patients with rheumatoïde arthritis. J Periodontol 2008;79:1689-96.

[38] Kaux JF, Forthomme B, Goff CL, Crielaard JM, Croisier JL. Current opinions on tendinopathy. J Sports Sci Med 2011;10:238-53.

[39] Kannus P1, Paavola M, Paakkala T, Parkkari J, Järvinen T, Järvinen M. Pathophysiology of overuse tendon injury. Radiologe 2002;42: 766-70.

[40] Smets F, Croisier JL, Forthomme B, Crielaard JM, Kaux JF. Applications cliniques du plasma riche en plaquettes (PRP) dans les lésions tendineuses. Sci Sports 2012;27:141-53.

[41] Kaux JF, Crielaard JM. Platelet-rich plasma application in management of chronic tendinopathies. Acta Orthop Belg 2013;79:10-5. 